\title{
Fractal-Based EEG Data Analysis of Body Parts Movement Imagery Tasks
}

\author{
Montri PHOTHISONOTHAI and Masahiro NAKAGAWA \\ Chaos and Fractals Informatics Laboratory, Nagaoka University of Technology, Nagaoka, Niigata 940-2188, Japan
}

\begin{abstract}
The objective of this study is to analyze the spontaneous electroencephalographic (EEG) data corresponding to body parts movement imagery tasks in terms of fractal properties. We proposed the six algorithms of fractal dimension (FD) estimators; box-counting algorithm, Higuchi algorithm, variance fractal algorithm, detrended fluctuation analysis, power spectral density analysis, and critical exponent analysis. The different parts of human body movement imagination such as feet, tongue, and index finger are proposed for use as the tasks in this experiment.
\end{abstract}

The EEG data were recorded from three healthy subjects (2 males and 1 female). The experimental results are useful in the measurement of FD changes in EEG data and present different characteristics in terms of variability. The probability density function (PDF) is also applied to show that the FD distribution is along each electrode. This study proposes that the performances of each method can extract information from the EEG data of imagined movement.

Key words: fractal dimension, electroencephalogram (EEG), brain wave, mental tasks, motor imagery task, EEG data analysis.

\begin{abstract}
An electroencephalogram (EEG) measures electrical activity from the neural currents of the billions of nerve cells within the human brain. The EEG data can be measured at the scalp surface through electrodes; this is called a noninvasive method. At present, an EEG is recognized as providing indispensable data to investigate brain functions. There are many researches applying the usefulness of EEG data, especially in the fields of medicine, applied physics, bioengineering, computer science, bioinformatics, and physiological science. For instance, EEG data applied to analyze the human brain $[1,2]$ and animal brain were also reported by Saito et al., Vincent et al., and Klerman et al. [3-5]. Furthermore, EEG data proposed for use as novel information in an interfacing technology between a human and a machine is called a brain-computer interface (BCI) [6]. This technology has been assisting not only healthy people, but also patients suffering from severe motor impairments and numerous other diseases for which more traditional methods cannot be used because only the cognitive functions remain intact. Significantly, such impairments affect more than 2 million people worldwide [7]. Recently, EEG data of imaginary movement (or mental tasks) have been proposed for use in systems in which the subjects imagine their motor movements (left or right hand) [8,9], and in basic natural
\end{abstract}

human decisions, i.e., "yes/no," has also been proposed $[10,11]$. Also, several recent studies in the analysis of EEG data are usually employing the fast Fourier transform (FFT). The FFT and correlation functions have been used frequently for a quantification of brain waves (EEG data). EEG data are composed of multiple-scale wave patterns with different kinds of local irregularities including periodic waves and paroxysmal waves. Thus their frequency spectra exhibit a $1 / f$-like profile, so the characterizing EEG data from a viewpoint of local irregularity using fractal theory might be useful [12]. In the past decade, the fractal dimension (FD) analysis of EEG data has been applied to various kinds of biomedical researches, such as the routine detection of dementia [12,13], EEG analysis of sleeping newborns [14], fractal spectral analysis of preepileptic seizures [15], and the clinical problems of acute stroke detection [16].

The use of FD analysis applied to EEG data was previously suggested by Boostani and Moradi, Popivanov et al., and Bashashati et al. [17-19]. However, these methods are applicable to investigating the imagination of hand movements and the extraction of visual information. In this article, we propose a possible improvement for the problem of binary communication by increasing the number of mental tasks up to four; however, so that we may af-

Received on Jun 11, 2007; accepted on Jul 13, 2007; released online on Jul 19, 2007; doi:10.2170/physiolsci.RP006307 Correspondence should be addressed to: Montri Phothisonothai, Chaos and Fractals Informatics Laboratory, Nagaoka University of Technology,1603-1 Kamitomioka, Nagaoka, Niigata, 940-2188 Japan. Tel: +81-258-47-1611 (Ext. 5146), Fax: +81-258-47-9500, Email: montri@pelican.nagaokaut.ac.jp, masanaka@vos.nagaokaut.ac.jp 
firm that the processing method is applicable to multistate BCI systems, the differences between the proposed mental tasks should be quantified and evaluated for each experimental subject. The proposed mental tasks in this experiment have not been previously analyzed through fractal techniques.

This article proceeds as follows. Section 1 discusses the EEG data acquisition. Section 2 presents the fractal analysis methods. Section 3 examines the experimental results. Section 4 discusses these results. Section 5 discusses the conclusion.

\section{EEG DATA ACQUISITION AND EXPERIMENTAL PARADIGM}

The electrodes were placed according to the 10-20 international system depicted in Fig. 1. The referencing and grounding electrodes were placed at the forehead and right earlobe of a subject. In this study, we are investigating the FD values on the sensorimotor area of the cortex because that area has an important influence on the motor imagery tasks [18, 19], i.e., movement imagination. Therefore the three electrode positions at C3, Cz, and C4 were selected. We tested the experimental paradigm on two males (SM1 and SM2) and one female (SF1). Their ages ranged from 21-32 (mean age 25.7 years); we collected the 80-trial data/subject (20-trial/task). During the experiments, they sat in a comfortable chair in an electrically shielded room, and the distance between the monitor and the subject's chair was about $1.0 \mathrm{~m}$. Four mental tasks

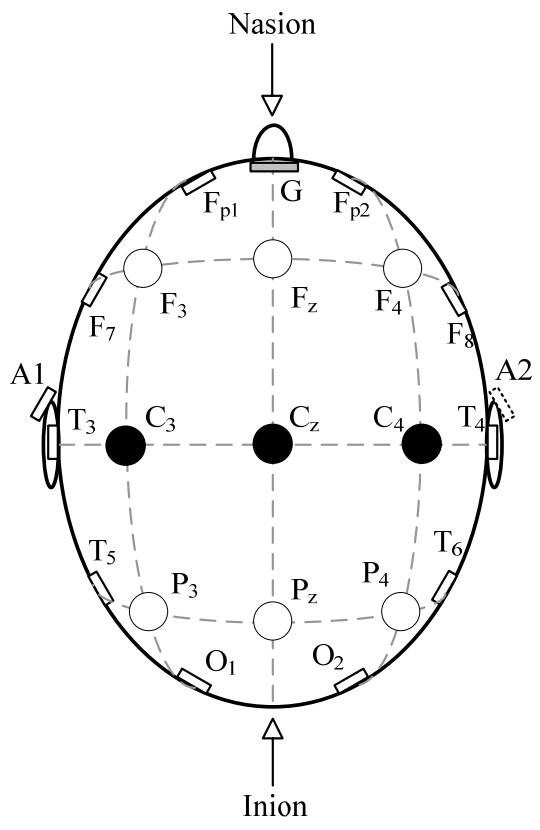

Fig. 1. The electrode positions of 3 channels that were placed on the scalp for EEG data acquisition is marked in black circles, C3, Cz, and C4. The grounding electrode is within a gray rectangle, $G$, and the referencing electrode is within a dotted rectangle, $\mathrm{A} 2$.

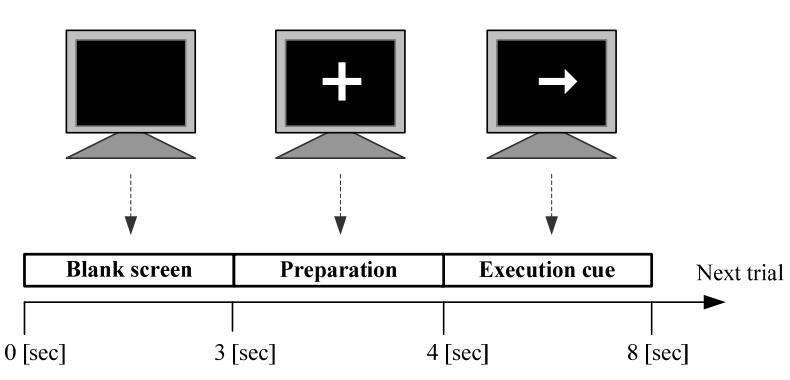

Fig. 2. One-trail epoch for a time sequence of the experiment with the indicators on a monitor.

were given to each subject for a one-trial period, as follows: (i) Imagine left index finger movement; (ii) Imagine right index finger movement; (iii) Imagine tongue movement; and (iv) Imagine feet movement. We set the one-trial epoch of one time period at $8 \mathrm{~s}$ throughout the experiment as depicted in Fig. 2, and the time period of the experiment was divided into three periods. In the first period, when the subjects had their eyes open on a blank screen, they would be in a relaxed state of mind (relaxed period). In the second period, the subjects were getting ready to perform the tasks while the cross "+" was displayed. In the third period, when they were viewing a screen showing the indicator, their mind would be in an active state of imagination (imagining period). To indicate whether tasks should be performed, the indicators were used as follows: the left arrow " $\leftarrow$ " was used for left index finger movement imagination, the right arrow " $\rightarrow$ " for right index finger movement imagination, the down arrow " $\downarrow$ " for feet movement imagination, and the horizontal line "_, for tongue movement imagination.

To acquire the EEG data, we used an EEG multiple channel amplifier (MEG-6116; Nihon Kohden, Tokyo, Japan) and an analog-to-digital converter (PC-CARDDAS16/16; Measurement Computing, MA, USA) in this experiment. An EEG electrode paste (Z-401CE; Nihon Kohden) was used for reduced impedance. The EEG data were digitized at 512 samples/s; resolution 16 bits/sample; and data were analog bandpass filtered from 0.5 to $100 \mathrm{~Hz}$.

\section{FRACTAL ANALYSIS}

Fractal geometry is a mathematical tool for dealing with complex systems. A method of estimating dimension has been widely used to describe objects in space, since it has been found useful for the analysis of biological data. Moreover, fractal dimension (FD) is one of most popular fractal features. The FD is relatively intensive to data scaling and shows a strong correlation with the human movement of EEG data $[17,18,20]$. The time series with fractal nature are to be describable by the functions of fractional Brownian motion ( $\mathrm{fBm}$ ), for which the FD can easily be set. Waveform FD values indicate the complexi- 
ty of a pattern or the quantity of information embodied in a waveform pattern in terms of morphology, spectra, and variance. The six algorithms have been proposed in the present study and were both in time and frequency domain analysis.

2.1. Box-counting method. One of the most common methods for calculating the FD of a self-similar fractal is the box-counting method (BCM). The definition of BCM is a bounded set in Euclidian $n$-space that it composes a self-similar property [21], $N_{r} \propto(1 / r)^{D}$, and by covering a structure with boxes of radii, $r$, the FD can be determined by

$$
D_{\mathrm{BCM}}=\lim _{r \rightarrow 0} \frac{\log _{2}\left(N_{r}\right)}{\log _{2}(1 / r)}
$$

We then repeat this process with several different radiuses. To implement this algorithm, the number of contained boxes, $N_{r}$, is computed from the difference between the maximum and minimum amplitudes of the data divided by the changed radius, as

$$
\begin{aligned}
& n_{r}(i)=\left\lfloor\frac{\max \left(x_{r}\right)-\min \left(x_{r}\right)}{r(i)}\right\rfloor \\
& \quad \text { for }\left\{r \in 2^{k} \mid k=1,2, \ldots, \log _{2}(L)-1\right\} \\
& N_{r}=\sum_{i} n_{r}(i)
\end{aligned}
$$

where $N_{r}$ is a total number of contained boxes, $x_{r}$ represents the EEG time series with length $L 2048$ points (we investigate the imagining period 4 to $8 \mathrm{~s}$ or 512 samples $\times$ $4 \mathrm{~s}), r(i)$ is a radius by changing a step of $k$ within the $i$-th subdivision window and an integer-part function denoted by [ $\cdot$ ]. To obtain the FD, the least-square linear fitted line corresponding to the slope of the plot $\log _{2}\left(N_{r}\right)$ versus $\log _{2}(1 / r)$ is applied.

2.2. Variance fractal dimension (VFD). This method is determined by the Hurst exponent, $H$, whose calculation was divided from the properties of the $\mathrm{fBm}$ data. The basic idea of calculation is based on the power law relationship between the variance of the amplitude increments of the input time series, which was produced by a dynamic process over time. The main advantage of VFD was its support of the real-time computation [22]. For example, the VFD was applied to lung-sound analysis [23] and fighting fish trajectory classification [24].

We also selected the VFD for estimating the FD of EEG data. The amplitude increments of a datum over a time interval $\Delta t$ adhere to the following power law relationship $\operatorname{Var}\left[x\left(t_{2}\right)-x\left(t_{1}\right)\right] \propto\left|t_{2}-t_{1}\right|^{2 H}$, and the Hurst exponent can be calculated by using a log-log plot, then given by

$$
H=\lim _{\Delta t \rightarrow 0}\left(\frac{1}{2} \cdot \frac{\log _{2}\left(\operatorname{Var}\left[(\Delta x)_{\Delta t}\right]\right)}{\log _{2}(\Delta t)}\right)
$$

The variance in each window per stage $k$ can be calculated as follows

$$
\begin{aligned}
& \operatorname{Var}\left[\Delta x_{\Delta t}\right]=\frac{1}{\left(N_{k}-1\right)}\left[\sum_{j=1}^{N_{k}}(\Delta x)_{j k}^{2}-\frac{1}{N_{k}}\left(\sum_{j=1}^{N_{k}}(\Delta x)_{j k}\right)^{2}\right] \\
& (\Delta x)_{j k}=x\left(j n_{k}\right)-x\left((j-1) n_{k}\right), \text { for } j=1,2, \ldots, N_{k}
\end{aligned}
$$

The least-square linear fitted line corresponds to the slope of the plot $\log _{2}\left(n_{k}\right)$ and $\log _{2}\left(\operatorname{Var}[\Delta x]_{k}\right)$, the Hurst exponent is computed as $H=0.5 s$, where $s$ is the obtained slope, and the FD can then be estimated as

$$
D_{\mathrm{VFD}}=2-H
$$

The process of calculating the FD essentially involves segmenting the entire input time series data into a subsequence (or window). The values $k$ represents are the integer range chosen so that each window of size $N_{T}$ contains a number $n_{k}=2^{k}$ of smaller windows of size $N_{k}=\left[N_{T} / n_{k}\right]$. According to the initial step, we ranged the step size of window $k=2,3, \ldots, 10$.

2.3. Higuchi method (HM). FD is another measure of data complexity, generally evaluated in phase space by means of correlation dimension. Higuchi proposed an algorithm for the estimation of FD directly in time domain without reconstructing the strange attractor [25]. The HM also gives a reasonable estimate of the FD in the case of a short time segment. The HM algorithm based on the given finite time series $y=\{y(1), y(2), \ldots, y(N)\}$, which is a new time series, $y_{m}^{k}$, is constructed by the following equation,

$$
\begin{aligned}
& y_{m}^{k}=\left\{y(m), y(m+k), y(m+2 k), \ldots, y\left(m+\left\lfloor\frac{N-m}{k}\right\rfloor\right) \cdot k\right\} \\
& \quad \text { for } m=1,2, \ldots, k
\end{aligned}
$$

where both $m$ and $k$ are integers and indicate the initial time and the time interval, respectively. The length, $L_{m}(k)$, of each curve is computed as

$$
L_{m}(k)=\frac{1}{k}\left\{\left(\sum_{i=1}^{\left\lfloor\frac{N-m}{k}\right\rfloor}|y(m+i k)-y(m+(i-1) k)| \cdot \frac{N-1}{\left[\frac{N-m}{k}\right] \cdot k}\right\}\right.
$$

The length of curve for time interval $k, L_{m}(k)$ is computed as the average of the $m$ curves. A relationship of this algorithm is $L_{m}(k) \propto k^{-D_{H M}}$; therefore we apply the leastsquares fitting line of $\log _{2} L[(k)]$ versus $\log _{2}(k)$, and the negative slope of the obtained line is calculated, giving the estimate of the FD and $D_{H M}$. In the experiment, we set $k_{\max }=2^{7}$.

2.4. Detrended fluctuation analysis (DFA). This method was initially proposed by Peng et al. [26]. The idea of DFA was invented originally to investigate the long-range dependence in coding and noncoding DNA nucleotide se- 
quence. Because of simplicity in its implementation, the DFA is now becoming the most important method in the field of fractal analysis [27]. Therefore the DFA method was also applied to FD estimation in this study.

$$
X(k)=\sum_{i=1}^{k}[x(i)-\bar{x}]
$$

This integrated series is divided into nonoverlapping intervals of length $n$. In each interval, a least-squares line is fit to the data (representing the trend in the interval). The series $X(k)$ is then locally detrended by subtracting the theoretical values $X_{n}(k)$ given by the regression. For a given interval length $n$, the characteristic size of fluctuation for this integrated and detrended series is calculated by

$$
F(n)=\sqrt{\frac{1}{N} \sum_{i=1}^{N}\left[X(k)-X_{n}(k)\right]^{2}}
$$

This computation is repeated over all possible interval lengths, $n$. In practice, the minimum length is ranged from 10 samples to a half-length of input data, giving two adjacent intervals. We set $n=2^{k}$ for $k=4,5, \ldots, \log _{2}(L)-1$ because the power of a 2 based length to input EEG data is used in this experiment. The relationship between the detrended series and interval lengths can be expressed as $F(n) \propto n^{\alpha}$, PSD can then be converted into the Hurst exponent $H=\alpha-1$ and the estimated FD accordingly as $D_{\mathrm{DFA}}=3-\alpha$ where $\alpha$ is expressed as the slope of a double logarithmic plot $\log _{2}[F(n)]$ versus $\log _{2}(n)$.

2.5. Power spectral density analysis. This method is widely used to assess the fractal properties of time series, the method based on frequency domain analysis, and it also works on the basis of the periodogram obtained by the fast Fourier transform (FFT) algorithm. It is called a power-law relationship of Mandelbrot and van Ness [28] and can be expressed as follows:

$$
f(s) \propto \frac{1}{s^{-\beta}}
$$

where $s$ is the frequency, $f(s)$ is the correspondence power at a given frequency, and $\beta$ is the scaling exponent, which is estimated by calculating the negative slope of the linear fit relating $\log f(s)$ to $\log s$. The power spectral density analysis (PSDA) was applied to raw EEG data to classify the EEG characteristics.

This study used the enhanced version of PSDA that initially was proposed by Fougère [29] and modified by Eke [30]. To improve the stability of spectral estimates, raw EEG data of each individual trial were first detrended by subtracting the mean of the data series from each value, and then each value is multiplied by a parabolic window, as follows:

$$
\psi(i)=1-\left(\frac{2 i}{M+1}-1\right)^{2} \text { for } i=1,2, \ldots, M
$$

The scaling exponent was estimated by the least-square fitting of log-log domain for frequencies lower than $1 / 8$ of the sampling rate, i.e., less than $64 \mathrm{~Hz}$ in this study. The Hurst exponent can be determined as $H=(\beta-1) / 2$ where $1<\beta<3$, and the estimated FD can be computed by the following equation

$$
D_{P S D A}=2+\frac{1-\beta}{2}
$$

2.6. Critical exponent method. This method was initially proposed by Nakagawa [31]. The critical exponent method (CEM) has been applied to the physiological data analysis, featured by Sabanal and Nakagawa, Petry and Barone, Petry et al., and Nakagawa [32-35]. The PSD of observed fBm data, $P_{H}(v)$, in the frequency domain is determined as

$$
P H(v) \propto v^{2 H+1}=v^{-\beta}
$$

The moment, $I_{\alpha}$, and the moment exponent, $\alpha$, of the PSD are determined as

$$
I_{\alpha}=\int_{0}^{\Omega} d v P_{H}(v) v^{\alpha},(-\infty<\alpha<\infty)
$$

We will consider the frequency bands as finite values and substitute Eq. 15 in Eq. 16; thus the equation was given as

$$
I_{\alpha} \propto \int_{1}^{\Omega} d v v^{\alpha-\beta}=\int_{1}^{\Omega} d v v^{A-1}=\frac{1}{A}\left(\Omega^{A}-1\right)=\frac{2}{A} \exp \left(\frac{u A}{2}\right) \sinh \left(\frac{u A}{2}\right)
$$

where $A=\alpha-\beta+1, u=\log \Omega$ and $\Omega$ is the frequency variable that was normalized to the lower bound of the integration region as 1 . Thus by taking the logarithm of moment, the third order derivative can be written as

$$
\frac{d^{3} \log I_{\alpha}}{d \alpha^{3}}=\frac{2}{8} u^{3} \operatorname{cosech}^{3}\left(\frac{u A}{2}\right) \cosh \left(\frac{u A}{2}\right)-\frac{2}{A^{3}}
$$

We then determine the critical value $\alpha=\alpha_{c}$, which satisfies for $d^{3} \log I_{\alpha} / d \alpha^{3}=0$. Finally, the FD can be estimated by

$$
D_{C E M}=2-\frac{\alpha_{c}}{2}
$$

The PSDA and CEM used the 2048-point FFT algorithm for transforming the EEG time series data into frequency components. To implement the CEM according to Eq. 18, we set the step size of the moment exponent, which had been $\alpha_{\Delta}=0.01$.

\section{EXPERIMENTAL RESULTS OF EEG DATA ANALYSIS}

During the experiments, we assign the acting stimuli to help the subject easily perform the tasks. The given acting stimuli for each of four motor imagery tasks were as follows: (i) Feet movement imagination (FT-MI); imagining up-down lifting; (ii) Left index finger movement imagi- 


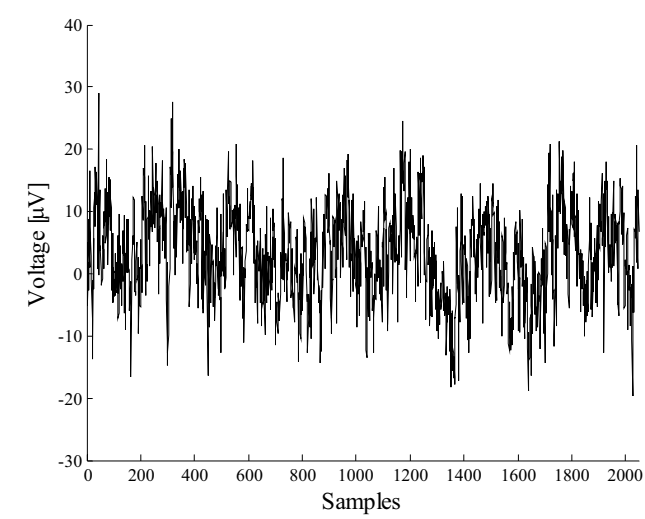

(a)

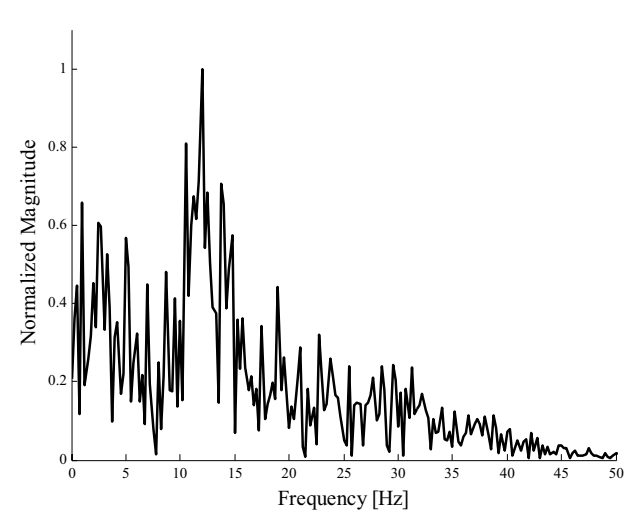

(b)

Fig. 3. A typical example of raw EEG data recorded from FTMI at the electrode C4 position. (a) Sampled EEG data waveform. (b) Power spectrum distribution of EEG data.

nation (LF-MI): imagine mouse click by using left index finger of left hand side; (iii) Right index finger movement imagination (RF-MI): imagine mouse click by using right index finger of right-hand side; (iv) Tongue movement imagination (TG-MI): imagine up-down movement.

To evaluate the FD of EEG data, the time period of imagined movement ( $4 \mathrm{~s}$ to $8 \mathrm{~s}$ ) in each trial was processed or 2,048 points (512 samples $\times 4 \mathrm{~s}$ ). A summary of the selected parameter values in this experiment as shown in Table I. To show the calculating step of each algorithm, we tested with the EEG data recorded from FT-MI, and the results of fractal analysis are shown in Figs. 3 to 5.

This article presents the fractal dimension algorithms based on both time and frequency domain approaches. The power spectrum can be used to study relationships across time scales. For instance, Fig. 4 shows the power spectrum of EEG data and time-axis scaling. As a linear fitted line on a log-log plot, the scaling exponent can be expressed as the slope $\beta=\lim \log f(s) / \log s$ with $\beta<0$ for imaging period. This means that the brain dynamics in an imaginary state have a frequency spectrum exhibiting a $1 /$ $f$-like profile characteristic. Figure $4 \mathrm{~b}$ shows time-axis scaling of the complexities in waveform patterns of a re-

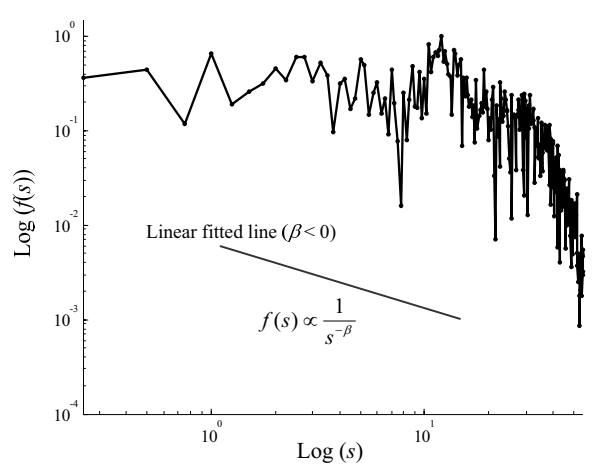

(a)
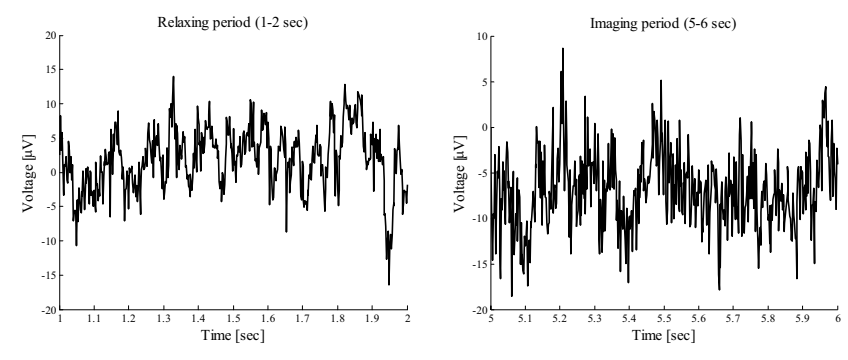

(b)

Fig. 4. Fractal concepts to characterize fluctuations of EEG data. (a) Power spectrum of EEG data in log-log plot. For $\beta<$ 0 , this scaling exponent of power laws exhibits long trends. (b) Comparing EEG data in time-axis scaling between relaxing and imaging periods.

laxing state lower than an imagining state. The important point here is that these scaling properties can be found not only in EEG activity [36] but also in imaginary activities. All fractal algorithms through selected parameters have been applied to analyzing the EEG data of four different motor imagery tasks. The results of estimated FDs from each subject are shown in Tables 2 through 7 . To show the variability of the fractal algorithms, we also examined FD values on the basis of statistical quantities that are the standard deviation (SD) and average values of all trial data. Also, we then examined the computation time, where we used a laptop with a $1.60 \mathrm{GHz} \mathrm{CPU}$ and $512 \mathrm{MB}$ of memory. The output average computation time for each method is shown in Fig. 6.

Table 1. Summary of selected parameter values.

\begin{tabular}{cll}
\hline Algorithm & \multicolumn{1}{c}{ Parameter } & \multicolumn{1}{c}{ Value } \\
\hline BCM & Step size of radius & $k=1,2, \ldots, 10$ \\
VFD & Step size of window & $k=2,3, \ldots, 10$ \\
HM & Maximum interval length & $k_{\max }=2^{7}$ \\
DFA & $\begin{array}{c}\text { Step size of interval } \\
\text { length }\end{array}$ & $k=4,5,6, \ldots, 10$ \\
PSDA & $\begin{array}{c}\text { Maximum frequency } \\
\text { index, FFT point }\end{array}$ & $s=64 \mathrm{~Hz}, N_{F F T}=2,048$ \\
& $\begin{array}{c}\text { FFT point, step size of } \\
\text { CEM }\end{array}$ & $N_{F F T}=2,048, \alpha_{\Delta}=0.01$ \\
& moment exponent \\
\hline
\end{tabular}




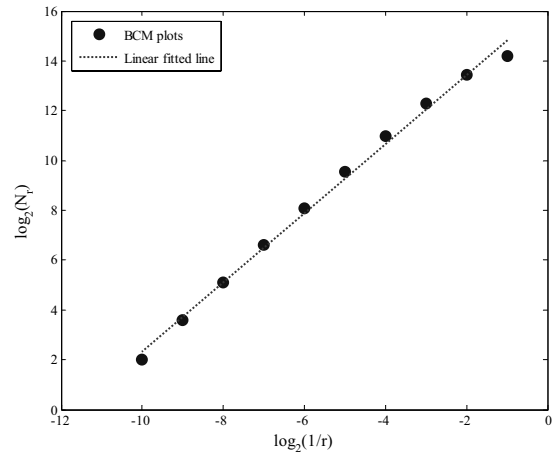

(a)

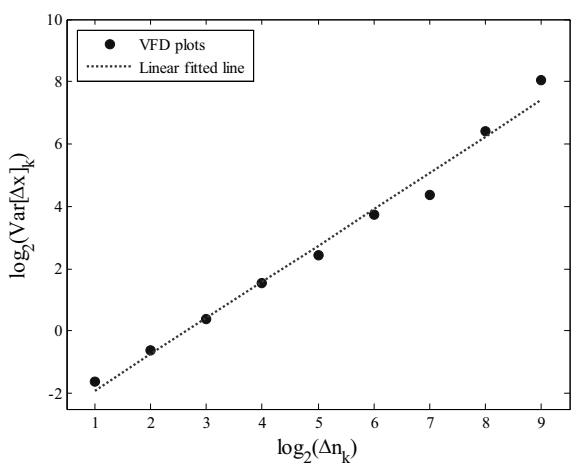

(b)

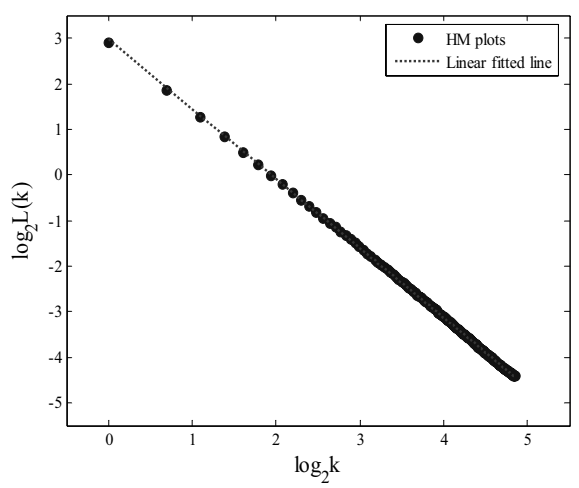

(c)

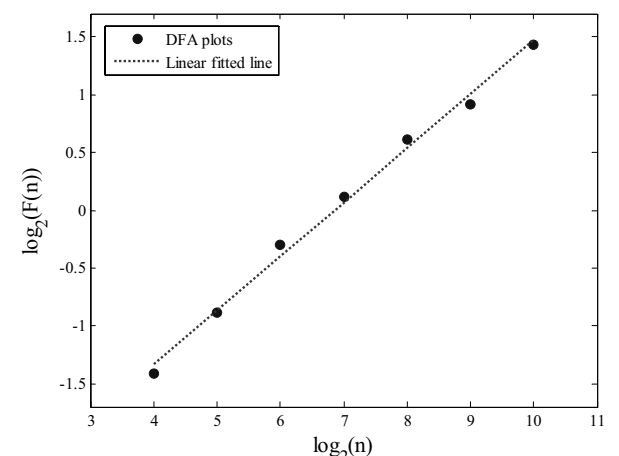

(d)

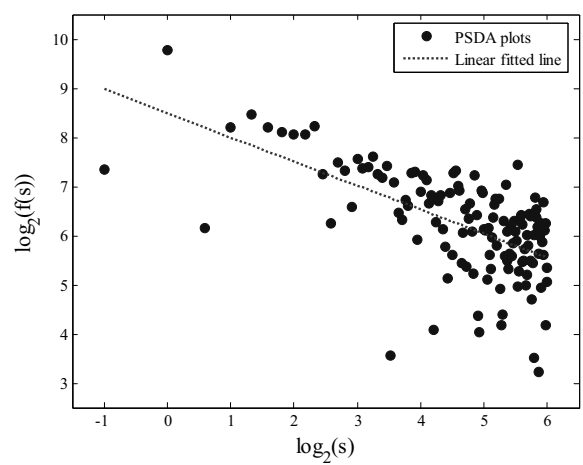

(e)

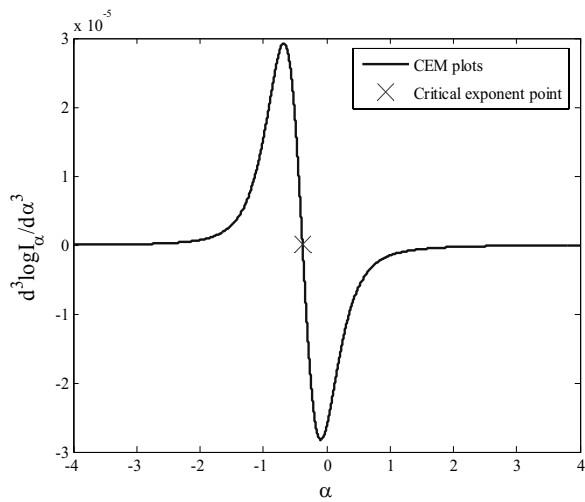

(f)
Fig. 5. The results for fractal analysis, performed on a series of test EEG data. (a) BCM algorithm: a loglog plot of a total number of contained boxes versus radius. (b) VFD algorithm: a log-log plot of variance versus window length. (c) HM algorithm: a log-log plot of a curve's length versus interval length. (d) DFA algorithm: a log-log plot of a total length $F(n)$ versus interval length. (e) PSDA algorithm: a log-log plot of power spectra versus frequency. (f) CEM algorithm: a log-log plot of moment function versus moment exponent value.

\section{DISCUSSION}

Based on the obtained results in Tables 2 through 7, all subjects show that the FD values of the proposed motor imagery tasks existed. The six algorithms present specific properties in terms of capability and variability. Some algorithms appeared inapplicable for the EEG time series data in this study, such as PSDA and CEM algorithms, for example. The main reason is that two algorithms based on frequency analysis since estimating the Hurst exponent can be utilized by means of the FFT method where the FFT can be substantially applicable in the long-time series data, and it easily declines when the EEG data has been analyzed in a short-time series. However, we can enhance the variability and avoid such drawbacks by in-

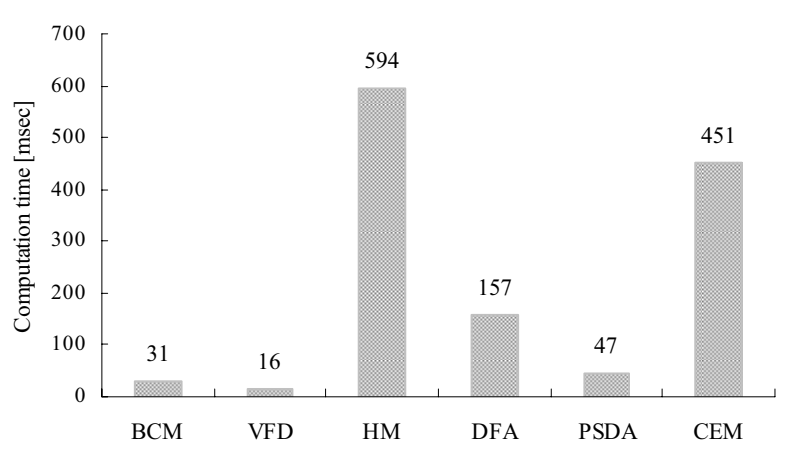

Fig. 6. The average computation time of the proposed algorithms in milliseconds.

creasing the sampling rate at the same period of experiment. In terms of variability, the HM algorithm presents the lowest average values of SD; we found that the SD is 
Table 2. BCM algorithm, the average values of FD and SD.

\begin{tabular}{|c|c|c|c|c|c|}
\hline \multirow{2}{*}{ Subject } & \multirow{2}{*}{ Electrode position } & \multicolumn{4}{|c|}{ Task } \\
\hline & & LF-MI & RF-MI & FT-MI & TG-MI \\
\hline \multirow{4}{*}{ SM1 } & C3 & 1.7550 & 1.7425 & 1.7528 & 1.7519 \\
\hline & $\mathrm{Cz}$ & 1.8053 & 1.7381 & 1.7613 & 1.7966 \\
\hline & $\mathrm{C} 4$ & 1.8356 & 1.7541 & 1.8062 & 1.7731 \\
\hline & (SD) & $( \pm 0.0125)$ & $( \pm 0.0132)$ & $( \pm 0.0121)$ & $( \pm 0.0144)$ \\
\hline \multirow{4}{*}{ SM2 } & C3 & 1.7891 & 1.7050 & 1.7247 & 1.7950 \\
\hline & $\mathrm{Cz}$ & 1.9444 & 1.7975 & 1.8622 & 1.9038 \\
\hline & $\mathrm{C} 4$ & 1.8606 & 1.7478 & 1.7925 & 1.8062 \\
\hline & (SD) & $( \pm 0.0258)$ & $( \pm 0.0256)$ & $( \pm 0.0154)$ & $( \pm 0.0170)$ \\
\hline \multirow{4}{*}{ SF1 } & $\mathrm{C} 3$ & 1.8519 & 1.7634 & 1.8181 & 1.7659 \\
\hline & $\mathrm{Cz}$ & 1.7953 & 1.7394 & 1.7997 & 1.7559 \\
\hline & $\mathrm{C} 4$ & 1.8550 & 1.7506 & 1.7850 & 1.7641 \\
\hline & (SD) & $( \pm 0.0165)$ & $( \pm 0.0167)$ & $( \pm 0.0195)$ & $( \pm 0.0167)$ \\
\hline
\end{tabular}

Table 3. VFD algorithm, the average values of FD and SD.

\begin{tabular}{cccccc}
\hline \multirow{2}{*}{ Subject } & \multirow{2}{*}{ Electrode position } & \multicolumn{3}{c}{ Task } \\
\cline { 2 - 6 } & $\mathrm{C} 3$ & LF-MI & RF-MI & FT-MI & TG-MI \\
\hline \multirow{2}{*}{ SM1 } & $\mathrm{Cz}$ & 1.7869 & 1.6906 & 1.7147 & 1.7437 \\
& $\mathrm{C} 4$ & 1.8338 & 1.7044 & 1.7163 & 1.8131 \\
& $(\mathrm{SD})$ & 1.8863 & 1.7159 & 1.7569 & 1.8053 \\
& $\mathrm{C} 3$ & $( \pm 0.0176)$ & $( \pm 0.0178)$ & $( \pm 0.0146)$ & $( \pm 0.0190)$ \\
\hline \multirow{2}{*}{$\mathrm{SM} 2$} & $\mathrm{Cz}$ & 1.8025 & 1.6747 & 1.6984 & 1.7650 \\
& $\mathrm{C} 4$ & 1.9647 & 1.7766 & 1.7328 & 1.8266 \\
& $\mathrm{SD})$ & $( \pm 0.0145)$ & $( \pm 0.0192)$ & 1.7650 & 1.8569 \\
& $\mathrm{C} 3$ & 1.8909 & 1.7663 & $1.0122)$ & $( \pm 0.0139)$ \\
\hline \multirow{2}{*}{$\mathrm{SF} 1$} & $\mathrm{Cz}$ & 1.8556 & 1.7097 & 1.7528 & 1.8547 \\
& $\mathrm{C} 4$ & 1.9056 & 1.7334 & 1.7284 & 1.8078 \\
& $\mathrm{SD})$ & $( \pm 0.0278)$ & $( \pm 0.0176)$ & $( \pm 0.0178)$ & $( \pm 0.0157)$ \\
\hline
\end{tabular}

Table 4. HM algorithm, the average values of FD and SD.

\begin{tabular}{|c|c|c|c|c|c|}
\hline \multirow{2}{*}{ Subject } & \multirow{2}{*}{ Electrode position } & \multicolumn{4}{|c|}{ Task } \\
\hline & & LF-MI & RF-MI & FT-MI & TG-MI \\
\hline \multirow{4}{*}{ SM1 } & C3 & 1.7709 & 1.7212 & 1.7078 & 1.7281 \\
\hline & $\mathrm{Cz}$ & 1.8261 & 1.8091 & 1.7131 & 1.8006 \\
\hline & $\mathrm{C} 4$ & 1.8503 & 1.8653 & 1.7322 & 1.7397 \\
\hline & (SD) & $( \pm 0.0107)$ & $( \pm 0.0106)$ & $( \pm 0.0112)$ & $( \pm 0.0117)$ \\
\hline \multirow{4}{*}{ SM2 } & C3 & 1.7897 & 1.8103 & 1.6747 & 1.7941 \\
\hline & $\mathrm{Cz}$ & 1.9300 & 1.8047 & 1.7706 & 1.8381 \\
\hline & C4 & 1.8381 & 1.8331 & 1.7631 & 1.8369 \\
\hline & (SD) & $( \pm 0.0121)$ & $( \pm 0.0110)$ & $\pm 0.0103)$ & $( \pm 0.0107)$ \\
\hline \multirow{4}{*}{ SF1 } & C3 & 1.8478 & 1.8597 & 1.7450 & 1.8116 \\
\hline & $\mathrm{Cz}$ & 1.8213 & 1.7963 & 1.7288 & 1.7209 \\
\hline & $\mathrm{C} 4$ & 1.8350 & 1.8266 & 1.7441 & 1.7222 \\
\hline & (SD) & $( \pm 0.0106)$ & $( \pm 0.0121)$ & $( \pm 0.0132)$ & $( \pm 0.0112)$ \\
\hline
\end{tabular}

less than $\pm 0.0117, \pm 0.0121$, and \pm 0.0132 for subjects SM1, SM2, and SF1, respectively. Further, other algorithms, for instance, the VFD and DFA, were also present for the reasonable results. To feature the FDs, we determined the PDF theoretically by normalized histograms to show how the distributed FD values are with the four mo- tor imagery tasks, and we then compared all estimated FDs; these results are shown in Figs. 7 to 9.

The obtained results of this study signify that the EEG data of imaginary tasks contain fractal structures whose characteristics can be distinguished from a statistical model as PDF. However, the subjects exhibited their own 
Table 5. DFA algorithm, the average values of FD and SD.

\begin{tabular}{cccccc}
\hline \multirow{2}{*}{ Subject } & \multirow{2}{*}{ Electrode position } & \multicolumn{3}{c}{ Task } \\
\cline { 3 - 6 } & & LF-MI & RF-MI & FT-MI & TG-MI \\
\cline { 3 - 6 } SM1 & C3 & 1.7178 & 1.6934 & 1.7503 & 1.6331 \\
& Cz & 1.7122 & 1.8019 & 1.8122 & 1.6231 \\
& C4 & 1.7441 & 1.7644 & 1.7959 & 1.6584 \\
(SD) & $( \pm 0.0146)$ & $( \pm 0.0136)$ & $( \pm 0.0146)$ & $( \pm 0.0173)$ \\
\hline \multirow{2}{*}{ SM2 } & C3 & 1.7022 & 1.8119 & 1.7806 & 1.6347 \\
& Cz & 1.7919 & 1.8641 & 1.8331 & 1.7034 \\
& C4 & 1.8106 & 1.8213 & 1.8056 & 1.7597 \\
SF1 & (SD) & $( \pm 0.0165)$ & $( \pm 0.0179)$ & $( \pm 0.0146)$ & $( \pm 0.0223)$ \\
& C3 & 1.7775 & 1.7481 & 1.7625 & 1.7378 \\
& Cz & 1.7769 & 1.6994 & 1.7381 & 1.7147 \\
& C4 & 1.8000 & 1.7112 & 1.7428 & 1.7206 \\
\end{tabular}

Table 6. PSDA algorithm, the average values of FD and SD.

\begin{tabular}{cccccc}
\hline \multirow{2}{*}{ Subject } & \multirow{2}{*}{ Electrode position } & \multicolumn{3}{c}{ Task } \\
\cline { 3 - 6 } & & LF-MI & RF-MI & FT-MI & TG-MI \\
\cline { 3 - 6 } SM1 & C3 & 1.7381 & 1.6609 & 1.6866 & 1.715 \\
& Cz & 1.8016 & 1.6841 & 1.6612 & 1.8719 \\
& C4 & 1.8319 & 1.7234 & 1.7294 & 1.8441 \\
& (SD) & $( \pm 0.0273)$ & $( \pm 0.0276)$ & $( \pm 0.0224)$ & $( \pm 0.0287)$ \\
\hline \multirow{2}{*}{ SM2 } & C3 & 1.7672 & 1.6528 & 1.6781 & 1.8059 \\
& Cz & 1.8634 & 1.715 & 1.7916 & 1.8781 \\
& C4 & 1.8394 & 1.7416 & 1.7594 & 1.8856 \\
SF1 & (SD) & $1 \pm 0.0201)$ & $( \pm 0.0278)$ & $( \pm 0.0186)$ & $( \pm 0.0252)$ \\
& C3 & 1.8172 & 1.7572 & 1.7197 & 1.9591 \\
& Cz & 1.8113 & 1.7569 & 1.7072 & 1.8322 \\
& C4 & $( \pm 0.0247)$ & $( \pm 0.0178)$ & $( \pm 0.0197)$ & $( \pm 0.0285)$ \\
\hline
\end{tabular}

Table 7. CEM algorithm, the average values of FD and SD.

\begin{tabular}{cccccc}
\hline \multirow{2}{*}{ Subject } & \multirow{2}{*}{ Electrode position } & \multicolumn{3}{c}{ Task } \\
\cline { 3 - 6 } & & LF-MI & RF-MI & FT-MI & TG-MI \\
\hline \multirow{2}{*}{ SM1 } & C3 & 1.7634 & 1.7622 & 1.8547 & 1.8478 \\
& Cz & 1.7394 & 1.7528 & 1.8078 & 1.8213 \\
& C4 & 1.7506 & 1.7284 & 1.7878 & 1.8350 \\
SM2 & (SD) & $( \pm 0.0189)$ & $( \pm 0.0215)$ & $( \pm 0.0178)$ & $( \pm 0.0189)$ \\
& C3 & 1.8122 & 1.7769 & 1.8438 & 1.9431 \\
& Cz & 1.8147 & 1.7759 & 1.8641 & 1.8719 \\
& C4 & 1.7594 & 1.7306 & 1.7647 & 1.8000 \\
SF1 & (SD) & $( \pm 0.0216)$ & $( \pm 0.0157)$ & $( \pm 0.0246)$ & $( \pm 0.0221)$ \\
& C3 & 1.7469 & 1.7316 & 1.7987 & 1.8219 \\
& Cz & 1.7241 & 1.7019 & 1.7253 & 1.7978 \\
& C4 & 1.8716 & 1.8228 & 1.8584 & 1.8734 \\
\end{tabular}

different FD features because the human brain did not similarly produce the EEG potentials, and it has a unique pattern of neuron pathways [37]. Therefore the differences between the proposed mental tasks were quantified and evaluated for each experimental subject. Moreover, as proposed algorithms we suggest that the most appropriate algorithm depends on an application purpose; for instance, the HM algorithm is suited for evaluating the FD of EEG data with high precision, whereas HM algorithm extremely consumed the processing time in comparison with the other algorithms. To improve the computation time, we can adapt the given parameters $k_{\max }$ as in Table 1; 


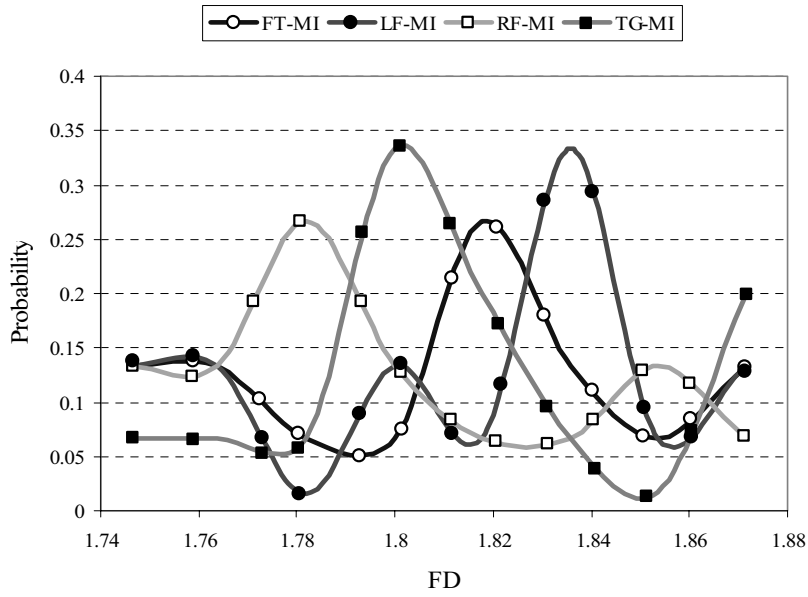

Fig. 7. A PDF of the four-motor imagery, subject SM1.

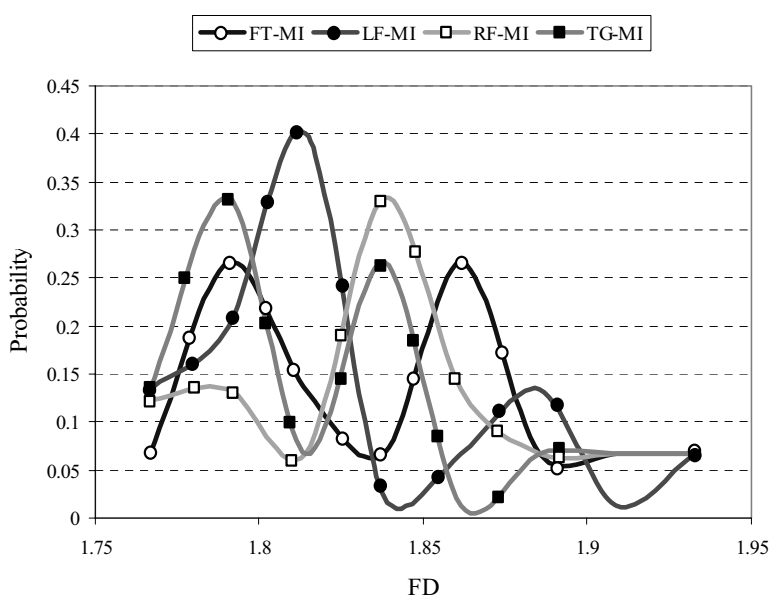

Fig. 8. A PDF of the four-motor imagery, subject SM2.

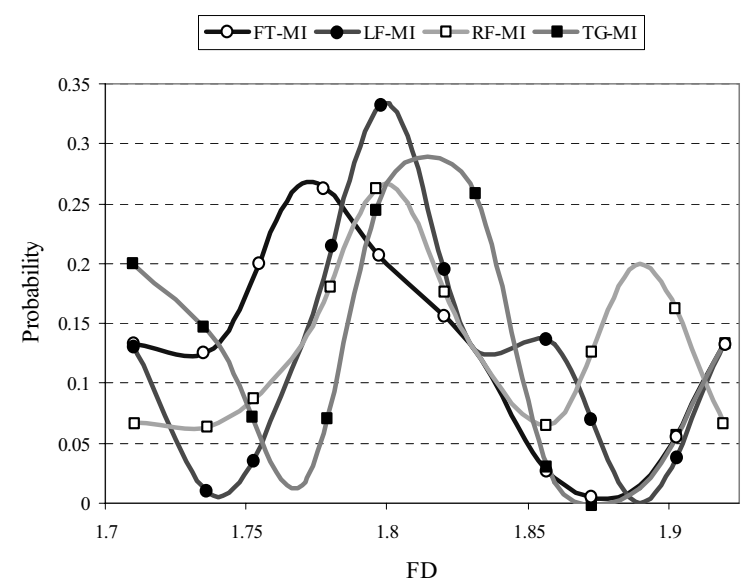

Fig. 9. A PDF of the four-motor imagery, subject SF1.

nevertheless, the expected variability would also not be identical. The VFD algorithm appropriates for real-time application, since its consumed computation time was merely $16 \mathrm{~ms}$.

\section{CONCLUSIONS}

The fractal analysis of EEG data of different motor imagery tasks has been proposed. We evaluated the FD of recorded EEG data from six fractal algorithms. The FD evaluation is a useful method for the analysis of time dependence of data in which the FD changes in EEG data. This study shows that human EEG possesses a self-affine property with a fractional dimension corresponding to the four motor imagery tasks. The chaotic behavior of EEG data presents a fractal temporal structure. Moreover, there is the evidence of the FD of mental tasks that imagined movements (FT-MI, RF-MI, LF-MI, and TG-MI) existed. The measurement of FD from EEG data can be capable not only in particular works, but also in publicity data. The FD characterizes the self-affine property of EEG data and has a direct relation to the different tasks.

This research was partially supported in part by the 21 st Century COE (Center of Excellence) Program "Global Renaissance by Green Energy Revolution" and the Grant-in-Aid for Scientific Research (15300070) from the Ministry of Education, Culture, Sports, Science and Technology of Japan. The authors would like to thank the subjects who took part in this research.

\section{REFERENCES}

1. Matsunami K, Homma S, Han X, Jiang Y. Generator sources of eeg large waves elicited by mental stress of memory recall or mental calculation. Jpn J Physiol. 2001:51:621-4

2. Hatta A, Nishihira Y, Kim SR, Kaneda T, Kida T, Kamijo K, Sasahara M, Haga S. Effects of habitual moderate exercise on response processing and cognitive processing in older adults. Jpn J Physiol. 2005;55:29-36.

3. Saito K, Suzuki H, Kawakami Y. Power spectrum density of eegs of sleeping epilepsy-prone el mice and their non-epileptic mother strain. J Physiol Sci. 2001;56:313-6.

4. Vincent JL, Patel GH, Fox MD, Snyder AZ, Baker JT, Van Essen DC, Zempel JM Snyder LH, Corbetta M, Raichle ME. Intrinsic functional architecture in the anaesthetized monkey brain. Nature. 2007:447: 83-6.

5. Klerman EB, Boulos Z, Edgar DM, Mistlberger RE, Moore-Ede MC. EEG delta activity during undisturbed sleep in the squirrel monkey. Sleep Res Online. 2000;3:113-9.

6. Mason SG, Birch GE. A general frame work for brain-computer interface. IEEE Neural Net Syst Rehab Eng. 2003:11:70-85.

7. Jonathan R, Wolpaw JR, Birbaumer N, McFarland DJ, Pfurtscheller G, Vaughan TM. Brain-computer interface for communication and control. Clin Neurophysiol. 2002;113:767-91.

8. Obermaier B, Muller GR, Pfurtscheller G. Virtual keyboard controlled by spontaneous eeg activity. IEEE Trans Neural Net Syst Rehab Eng. 2003;11:4226.

9. Millán JR, Renkens F, Mouriño J, Gerstner W. Brain-actuated interaction. Artif Intell. 2004;159:241-59.

10. Phothisonothai M, Nakagawa M. EEG-based classification of new imagery tasks using three-layer feedforward neural network classifier for brain-computer interface. J Phys Soc Jpn. 2006;doi:10.1143/jpsj.75.104801.

11. Kulish V, Sourin A, Sourina O. Human electroencephalograms seen as fractal time series: mathematical analysis and visualization. Com Biol Med. 2006:36:291-302.

12. Saji $R$, Konno H. Dynamical feature of the local fractal dimension of brain waves and its applicability for diagnosis of senile dementia. Jpn J Appl Phys. 2000;39:679-84.

13. Henderson G T, Ifeachor E C, Wimalaratna H S K, Allen T, Hudson R. Prospects for routine detection of dementia using the fractal dimension of the human electroencephalogram. Sci Meas Tech IEE Proc. 2000;147:321-6.

14. Accardo A P, Affinito M, Carrozzi M, Cisint S, Bouquet F. Comparison between spectral and fractal EEG analyses of sleeping newborns. IEEE Proc Eng Med 


\section{PHOTHISONOTHAI and M. NAKAGAWA}

Bio Soc. 1998:3:1569-71

15. Li X, Polygiannakis J, Kapiris P, Paratzakis A, Yao X. Fractal spectral analysis of pre-epileptic seizures in terms of criticality. J Neural Eng. 2005;2:11-6.

16. Hwa R C, Ferree T.C. Scaling properties of fluctuations in the human electroencephalogram. Phys Rev E. 2002; doi: 10.1103/physreve.66.021901.

17. Boostani R, Moradi MH. A new approach in the $\mathrm{BCl}$ research based on fractal dimension as feature and Adaboost as classifier. J Neural Eng. 2004;1:212-7.

18. Popivanov D, Jivkova S, Stomonyakov V, Nicolova G: Effect of independence component analysis on multifractality of eeg during visual-motor task. Sig Pro. 2005;85:2112-23

19. Bashashati A, Ward RK, Birch GE, Hashemi MR, Khalizadeh MA. Fractal dimension-based eeg biofeedback system. IEEE Proc Eng Med Bio Soc 2003:3:2220-3.

20. PreißI H, Lutzenberger W, Pulvermüller F, Birbaumer N. Fractal dimensions of short eeg time series in humans. Neurosci Lett. 1997;225:77-80.

21. Mandelbrot BB. The fractal geometry of nature. New York: W.H. Freeman; 1983.

22. Kinsner W: Batch and real-time computation of a fractal dimension based on variance of a time series. Univ Manitoba Canada: Tech Report. 1994;del94-6.

23. Gnitecki J, Moussavi Z. The fractality of lung sounds: a comparison of three waveform fractal dimension algorithms. Chaos Soli Frac. 2005;26:1065-72.

24. Kinsner W, Cheung V, Cannons K, Pear J, Martin T. Signal classification through multifractal analysis and complex domain neural network. IEEE Sys Man Cyb. 2006;36:196-203

25. Higuchi T. Approach to an irregular time series on the basis of the fractal theory. Physica D 1988; 31:277-83.

26. Peng CK, Mietus J, Hausdorff JM, Havlin S, Stanley HE, Goldberger AL. Long- range anticorrelations and non-Gaussian behavior of the heartbeat. Phys Rev Lett. 1993;70:1343-6.

27. Gu GF, Zhou WX. Detrended fluctuation analysis for fractals and multifractals in higher dimensions. Phys Rev E. 2006;doi:10.1103/physreve.74.061104.

28. Mandelbrot BB, Van Ness JW. Fractional Brownian motions, fractional noises and applications: SIAM Rev. 1968;10:422-37.

29. Fougère PF. On the accuracy of spectrum analysis of red noise processes using maximum entropy and periodogram methods: simulation studies and application to geographical data. J Geograp Res. 1985;90(A5):4355-66.

30. Eke A, Hermann P, Kocsis L, Kozak LR. Fractal characterization of complexity in temporal physiological signals. Physio Meas. 2002;23:R1-38.

31. Nakagawa M. A critical exponent method to evaluate fractal dimension of selfaffine data. J Phys Soc Jpn. 1993; doi:10.1143/jpsj.62.4233.

32. Sabanal S, Nakagawa M. The fractal properties of vocal sound and their application in the speech recognition model. Chaos Soli Frac. 1996;7:1825-43.

33. Petry A, Barone DAC. Fractal dimension applied to speaker identification. IEEE ICASSP'01. 2001:1:405-8.

34. Petry A, Augusto D, Barone C. Speaker identification using nonlinear dynamical features. Chaos Soli Frac. 2002;13:221-31.

35. Nakagawa M. Chaos and fractals in engineering. Singapore: World Sciencetific; 1999. p. 1-23.

36. Le Van Quyen M. Disentangling the dynamic core: a research program for neurodynamics at the large-scale. Biol Res. 2003;36:67-88.

37. Carran E, Stokes M. Learning to control brain activity: a review of the production and control of eeg components for driving brain-computer interface $(\mathrm{BCl})$ systems. Brain Cogn. 2003;51:326-36. 